\title{
Estudio cinético del proceso de biometanización de alpechín de almazara clásica previamente sometido a tratamiento aerobio con Geotrichum candidum
}

\author{
Por R. Borja Padilla*, A. Martín Martín ${ }^{* *}$ y M.M. Durán Barrantes*. \\ * Instituto de la Grasa y sus Derivados. Avda. Padre García Tejero, 4. 41012-SEVILLA. \\ ** Departamento de Ingeniería Química. Facultad de Ciencias, Avda. San Alberto Magno, s/n. 14004-CORDOBA
}

\section{RESUMEN}

Estudio cinético del proceso de biometanización de alpechín de almazara clásica previamente sometido a tratamiento aerobio con Geotrichum candidum

Se ha determinado la constante cinética aparente del proceso de digestión anaerobia de alpechín procedente de almazara clásica de prensas, que previamente se somete a un proceso de digestión aerobia con Geotrichum candidum dado su alto contenido en materia orgánica (DQO). Los resultados obtenidos ponen de manifiesto una acusada disminución de la misma (1-0,2 dias -1$)$ al aumentar la DQO $\left(6-11 \mathrm{~g} \mathrm{O}_{2} / 1\right)$ tanto en presencia de biomasa libre como soportada sobre Sepiolita (Pansil), lo que demuestra la existencia de un proceso de inhibición que se cuantifica utilizando el modelo cinético de Levenspiel.

La digestión anaerobia es capaz de eliminar el $80 \%$ de la DQO presente con un coeficiente de rendimiento medio de $315 \mathrm{ml} \mathrm{CH}_{4}$ en condiciones estándar de presión y temperatura/g DQO.

PALABRAS-CLAVE: Alpechín de prensa - Biometanización - Inhibición por sustrato - Modelo cinético - Pretratamiento aerobio.

\section{SUMMARY}

Kinetic study of the biomethanization of olive mill wastewater previously subjected to aerobic treatment with Geotrichum candidum

The apparent kinetic constant was determined for the anaerobic digetion of olive mill wastewater (OMW) which comes from clasical press factory previously subjected to aerobic digestion with Geotrichum candidum in view of his high content in organic matter. The results obtained revealed it to markedly decrease (from 1 to 0,2 days $^{-1}$ ) with increase in its COD (from 6 to $11 \mathrm{~g} / 1$ ) in the presence of both free and supported on Sepiolite (Pansil) biomass. This shows the existence of an inhibition process which is determined by using the Levenspiel kinetic model.

Anaerobic digestion allowed up to $80 \%$ of the COD present to be removed with an average yield coefficient of $315 \mathrm{ml} \mathrm{CH}_{4}$ STP/g COD.

KEY-WORDS: Aerobic pretreatment - Biomethanation - Classical olive mill wastewater - Kinetic model - Substrate inhibition.

\section{INTRODUCCION}

Entre las contaminaciones medioambientales que sufre la Cuenca Mediterránea destaca por su importancia y gravedad la provocada por las aguas residuales del proceso de obtención del aceite de oliva o alpechines, cuyo volumen asciende a 10 millones de $\mathrm{m}^{3} / a n ̃ o$, de los que 2 millones corresponden a España (Fiestas, J.A. y Borja, R., 1988). Este agua residual está constituida por el agua de vegetación de la aceituna a la que se le unen las aguas del lavado y procesado de las mismas; contiene además tejidos blandos de la pulpa y aceite emulsionado. Su composición cuantitativa es muy variable dependiendo principalmente del tipo de proceso empleado en la obtención del aceite, ya que mientras en los sistemas clásicos o de prensas la adición de agua es muy pequeña o nula, en los sistemas continuos se utiliza del orden de 1 litro de agua $/ \mathrm{kg}$ de aceituna. Presentan, por un lado un alto poder contaminante, y por otro una elevada actividad antibacteriana debida a la presencia de diversos compuestos fenólicos, lo que dificulta los distintos procesos aplicables para su depuración (De Caro, M. and Ligori, M.G., 1959) (Ragazzi, E. and Veronesse, G., 1967) (Sorlini, C. et al., 1959) (Rodríguez, M. et al., 1988).

Tabla I

Características del alpechín original y del alpechín fermentado de forma aerobia con Geotrichum candidum utilizado como influente en el proceso de digestión anaerobia.

\begin{tabular}{|c|c|c|}
\hline Parámetro & $\frac{\text { Alpechín }}{\text { original }}$ & $\frac{\text { Alpechín }}{\text { fermentado }}$ \\
\hline $\mathrm{pH}$ & 4,9 & 5,7 \\
\hline DQO (Demanda Química Oxígeno) & 90,0 & 45,0 \\
\hline Sólidos totales & 97,0 & 49,5 \\
\hline Sólidos totales minerales & 15,2 & 10,4 \\
\hline Sólidos totales volátiles & 81,8 & 39,1 \\
\hline Sólidos suspensión totales & 4,8 & 3,5 \\
\hline Sólidos suspensión minerales & 1,2 & 0,7 \\
\hline Sólidos suspensión volátiles & 3,6 & 2,8 \\
\hline Acidez volátil (ácido acético) & 0,5 & 0,3 \\
\hline Alcalinidad $\left(\mathrm{C}_{\mathrm{a}} \mathrm{CO}_{3}\right)$ & 1,8 & 1,4 \\
\hline Fenoles totales (ácido cafeico) & 1,9 & 1,1 \\
\hline Ortodifenoles (ácido cafeico) & 0,2 & 0,1 \\
\hline
\end{tabular}

Todas las concentraciones expresadas en $\mathrm{g} / \mathrm{l}$

La elevada concentración en materia orgánica existente en el alpechín procedente de almazara clásica o de prensa, hace que sus valores de las demandas química 
y biológica de oxígeno (DQO y DBO) lleguen a duplicarse respecto de los obtenidos en los sistemas continuos de centrifugación (Tabla I); ello hace necesario la utilización de varios procesos sucesivos para llegar a eliminar la mayor parte de la DQO existente en este tipo de alpechín.

Con la finalidad de desarrollar un proceso para la depuración de este alpechín de tan elevado poder contaminante se estudia la posibilidad de eliminar una parte de la fracción orgánica mediante fermentación aerobia con Geotrichum candidum y someter al residuo no biodegradado por este hongo a una digestión anaerobia, al presentar una carga orgánica más baja, óptima para el desarrollo del proceso. Este trabajo tiene dos objetivos; el primero consiste en la determinación de la constante cinética aparente del proceso de digestión anaerobia del efluente resultante del tratamiento aerobio citado anteriormente, y el segundo estudiar la influencia, que ejerce sobre la cinética, la inmovilización de las bacterias responsables del proceso anaerobio sobre un soporte de tipo arcilloso.

\section{PARTE EXPERIMENTAL}

\subsection{Proceso primario de tratamiento aerobio}

El alpechín utilizado, cuyas características se muestran en la Tabla I, se somete en primer lugar a un proceso de tratamiento aerobio con Geotrichum candidum. Para ello se utiliza un cultivo puro de esta especie fúngica, aislado del propio alpechín, procedente de la colección existente en el Centro de Estudios Biológicos (CSIC) de Madrid (Referencia IJFMA 534).

El proceso se realiza en fermentadores de un litro de volumen en régimen discontinuo durante tres días, a la temperatura de $25^{\circ} \mathrm{C}$, utilizando un caudal de aire de 1 litro/hora litro de alpechín, suministrado a través de difusores cerámicos.

Las características del alpechín una vez fermentado de esta forma se recogen en la Tabla I.

\subsection{Proceso secundario de depuración anaerobia}

\subsubsection{Equipo de digestión anaerobia}

El equipo usado consiste en unidades de digestión anaerobia (UDA), agitadas magnéticamente, de 1 litro de capacidad sumergidas en un baño de agua a $35^{\circ} \mathrm{C}$. El biogas generado pasa a través de una solución de $\mathrm{NaOH}$ para retener el $\mathrm{CO}_{2}$ producido. El volumen de metano se determinó indirectamente mediante la cantidad de agua desplazada por el gas (Borja, R. et al., 1990).

Se utilizaron dos equipos análogos al descrito, en uno de ellos los microorganismos estaban en suspensión (testigo) en tanto que en el otro se soportaron sobre un material arcilloso denominado Sepiolita (Pansil).

\subsubsection{Procedimiento experimental}

Como alimento de los digestores se utiliza el alpechín residual resultante del proceso de digestión aerobia con Geotrichum candidum, cuyas características se presentan en la Tabla I.
El proceso de digestión se inicia utilizando como inóculo una biomasa anaerobia previamente diluida y neutralizada procedente de una balsa de evaporación y lagunaje de alpechín.

A las UDAs se añaden inicialmente $750 \mathrm{ml}$ de agua destilada, $250 \mathrm{ml}$ de inóculo y $10 \mathrm{~g}$ de soporte en su caso. Tras un período de aclimatación de varios meses, los experimentos se llevan a cabo añadiendo 40,60, 80, 100,120 y $140 \mathrm{ml}$ de volumen de este líquido residual. Las cargas se añaden después de retirar el mismo volumen de líquido del biorreactor, tras decantar durante dos horas para evitar pérdidas de biomasa.

En cada experimento se mide el volumen de metano producido por día, así como la DQO inicial y final. La duración del experimento se corresponde con el tiempo necesario para la biometanización completa de cada carga. Todos los experimentos se realizaron por duplicado.

Los análisis de los parámetros de caracterización de los residuos se realizan de acuerdo con el "Standard Methods for the Examination of Water and Wastewater" (American Public Health Association, 1985).

Tabla II

Volumen de metano acumulado ( $\mathrm{ml}$ ) en función del tiempo (dias) para los distintos volúmenes de alimentación utilizados ( $\mathrm{ml}$ ). Digestor testigo

\begin{tabular}{crrrrrr} 
& \multicolumn{8}{c}{ Volumen alimentación } \\
Tiempo & 40 & 60 & 80 & 100 & 120 & 140 \\
\hline 1 & 285 & 375 & 330 & 300 & 325 & 305 \\
2 & 395 & 590 & 520 & 505 & 685 & 615 \\
3 & 435 & 765 & 706 & 635 & 1060 & 870 \\
4 & 450 & 825 & 866 & 737 & 1223 & 1150 \\
5 & 452 & 885 & 1001 & 897 & 1402 & 1315 \\
6 & - & 913 & 1095 & 949 & 1575 & 1490 \\
7 & - & 915 & 1191 & 1257 & 1650 & 1610 \\
8 & - & - & 1253 & 1350 & 1720 & 1705 \\
9 & - & - & - & - & 1760 & 1795 \\
10 & - & - & - & - & - & 1840 \\
11 & - & - & - & - & - & 1860 \\
12 & - & - & - & - & - & 1868 \\
13 & - & - & - & - & - & 1872
\end{tabular}

Tabla III

Volumen de metano acumulado ( $\mathrm{ml}$ ) en función del tiempo (dlas) para los distintos volúmenes de alimentación utilizados (ml). Soporte: Sepiolita "Pansil"

\begin{tabular}{crrrrrrr} 
& \multicolumn{8}{c}{ Volumen alimentación } \\
Tiempo & 40 & 60 & 80 & 100 & 120 & 140 \\
\hline 1 & 310 & 410 & 480 & 455 & 365 & 315 \\
2 & 410 & 691 & 870 & 865 & 715 & 651 \\
3 & 440 & 846 & 1105 & 1160 & 1110 & 990 \\
4 & 460 & 950 & 1207 & 1310 & 1325 & 1262 \\
5 & 465 & 980 & 1275 & 1375 & 1505 & 1502 \\
6 & - & 990 & 1320 & 1458 & 1660 & 1698 \\
7 & - & 995 & 1357 & 1479 & 1792 & 1841 \\
8 & - & - & 1362 & 1489 & 1792 & 1950 \\
9 & - & - & - & - & 1820 & 2021 \\
10 & - & - & - & - & - & 2074 \\
11 & - & - & - & - & - & 2110 \\
12 & - & - & - & - & - & 2140 \\
13 & - & - & - & - & - & 2142
\end{tabular}




\section{RESULTADOS EXPERIMENTALES}

En las Tablas II y III se presentan los volúmenes acumulados de metano en función del tiempo para los distintos volúmenes de alimentación utilizados en los dos digestores empleados.

Tabla IV

Evolución de la DQO (g/l) para los digestores Testigo $y$ con soporte Pansil

\begin{tabular}{ccccc} 
Carga $(\mathrm{ml})$ & \multicolumn{2}{c}{$D Q O_{\text {inicial }}$} & \multicolumn{2}{c}{$D Q O_{\text {final }}$} \\
\cline { 2 - 5 } & Testigo & Pansil & Testigo & Pansil \\
\hline 40 & 6,2 & 5,9 & 4,8 & 4,5 \\
60 & 7,2 & 6,9 & 5,7 & 5,0 \\
80 & 8,8 & 8,2 & 5,9 & 5,2 \\
100 & 9,8 & 9,2 & 5,8 & 5,4 \\
120 & 10,5 & 10,1 & 5,9 & 5,3 \\
140 & 11,4 & 10,9 & 5,9 & 5,4
\end{tabular}

La Tabla IV recoge los valores iniciales y finales de la DQO de los experimentos realizados, en ambos digestores.

\section{DISCUSION DE LOS RESULTADOS}

Mediante el tratamiento aerobio del alpechín utilizado, previo al proceso de digestión anaerobia, se consigue degradar el $50 \%$ de la DQO presente en este residuo (Tabla I), con lo que se obtiene un líquido residual con una carga orgánica más adecuada para llevar a cabo el proceso anaerobio. Por otro lado, su contenido en compuestos fenólicos queda reducido en un $42 \%$, con lo que de esta manera queda disminuido su poder antimicrobiano, aunque no obstante permanece aún una concentración relativamente elevada de estos compuestos $(1,1 \mathrm{~g} / \mathrm{l}$ en ácido cafeico).

El digestor anaerobio se puede considerar como un biorreactor en donde la materia orgánica, expresada como DQO, reacciona con los lodos microbianos de concentración X. La velocidad de eliminación de sustancia orgánica viene dada por (Winkler, M., 1983):

$$
(-d S / d t)=K S X
$$

donde $t$ es el tiempo, $\mathrm{K}$ un coeficiente cinético y $\mathrm{S}$ es la concentración de sustrato expresada en g DQO/. Dado el pequeño valor del coeficiente de rendimiento celular, $Y_{x / s}$, en la fermentación anaerobia (Grujer, W. and Zehnder, J.B., 1983), y considerando que la variación de la DQO en el transcurso de un experimento es reducida, se puede admitir que $X$ es, aproximadamente, constante. De hecho esta hipótesis se cumple para los dos digestores y en todos los experimentos ya que este parámetro se mantiene constante en el valor de $5,1 \mathrm{~g}$ de sólidos en suspensión volátiles/litro. Integrando (1) con esta condición se tiene:

$$
S=S_{0} \exp (-K X t)
$$

Definiendo un coeficiente de rendimiento $\left(\mathrm{Y}_{\mathrm{p} / \mathrm{s}}\right)$ para el producto formado (metano):

$$
Y_{p / s}=-d G / d S
$$

se tiene, finalmente:

$$
G=G_{m}\left[1-\exp \left(-K_{A} t\right)\right]
$$

donde $G$ es el volumen acumulado de metano, $G_{m}$ es el volumen acumulado a tiempo infinito; $G_{m}=S_{0} Y_{p / s}$, siendo $S_{o}$ la concentración inicial de sustrato y $K_{A}$ es una constante cinética aparente, $\mathrm{K}_{\mathrm{A}}=\mathrm{KX}$.

A partir de los datos contenidos en las Tablas II y III y utilizando un software standard (Time Series Processor, USA) se han calculado los parámetros $G_{m}$ y $K_{A}$. En la figura 1 se representa $K_{A}$ frente a la DQO inicial de los digestores en los dos sistemas estudiados; se observa que, en ambos casos, existe una acusada disminución de la velocidad del proceso al aumentar la DQO del medio; por otra parte, la inmovilización de los microorganismos no conduce a un comportamiento diferenciado ya que todos los puntos se agrupan en torno a una sola curva. Se puede observar que, a pesar de haber sometido el alpechín a un proceso previo de digestión aerobia, aún presenta carácter inhibidor. Experimentos anteriores (Fiestas, J.A. et al., 1990) llevados a cabo con alpechín procedente de almazara continua sin tratamiento aerobio, pusieron de manifiesto este mismo fenómeno, pero con carácter más acentuado.

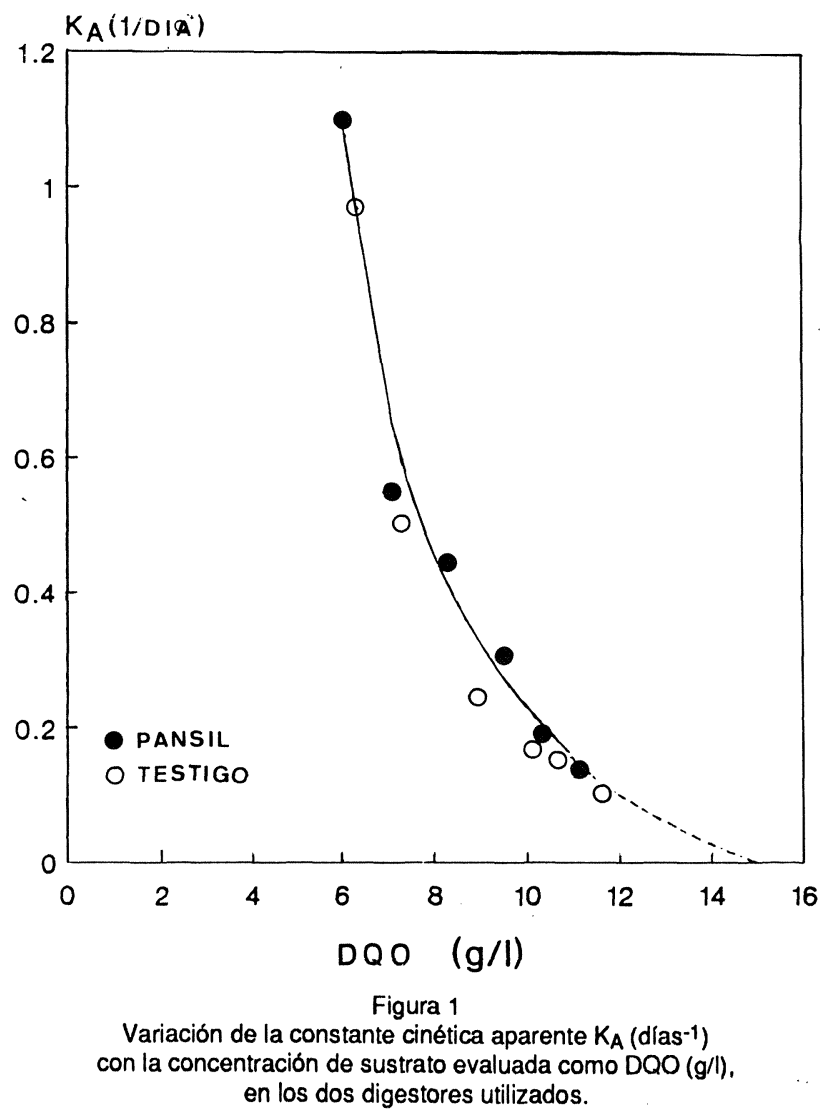

Con objeto de cuantificar la magnitud del proceso de inhibición provocado por el sustrato se utiliza el modelo 
propuesto por Levenspiel (Han, K. and Levenspiel, O., 1988):

$$
K_{A}=K_{0}\left[1-\left(S / S^{*}\right)\right]^{n}
$$

donde $K_{0}$ es la constante cinética aparente en ausencia de inhibición, $S^{*}$ es la concentración de inhibición crítica y $n$ es una constante adimensional que caracteriza la intensidad de la inhibición. Dado que $K_{A}$ varía con la concentración del sustrato, los valores representados en la figura 1 son valores medios dentro del intervalo de concentraciones de un experimento, lo que dificulta la obtención de las constantes $K_{0}$ y $n$. Determinando el valor medio de la función definida por (5) en el intervalo de concentraciones de un experimento dado se tiene:

$$
K_{A}=\frac{\left[\left(1-\left(S_{i} / S^{*}\right)\right)^{n+1}-\left(1-\left(S_{f} / S^{*}\right)\right)^{n+1}\right] K_{0} S^{*} \quad(6)}{\left(S_{f}-S_{i}\right)(n+1)}
$$

donde $S_{i}$ y $S_{f}$ representan la concentración inicial y final de un experimento, expresadas como DQO (tabla IV), y $\mathrm{K}_{\mathrm{A}}$ es la constante cinética aparente media para el mismo. Para la determinación de los parámetros $K_{0}$ y $n$ a partir de la ecuación (6), se utiliza el software de cálculo ya mencionado (Time Series Processor), teniendo en cuenta que el valor de $S^{*}$ (concentración crítica de inhibición) se puede determinar por extrapolación a $K_{A}=0$ de la ecuación $K_{A}=f(S)$ (Figura 1), al ser el valor de la concentración de sustrato para el cual la reacción o actividad de las células se detiene $\left(S^{*}=15 \mathrm{~g} / \mathrm{l}\right)$.

De esta forma se obtiene:

$$
\begin{aligned}
& \left(\mathrm{K}_{\mathrm{A}}\right) \text { Testigo }=17,5[1-(\mathrm{S} / 15)] 6,4 \\
& \left(\mathrm{~K}_{\mathrm{A}}\right) \text { Pansil }=14,3[1-(\mathrm{S} / 15)]^{6,2}
\end{aligned}
$$

Dada la proximidad de los valores de las constantes, el comportamiento de ambos tipos de digestores es análogo; por otra parte, el alto valor de $n$ en ambos casos $(n>6)$ indica la existencia de una fuerte inhibición, si bien en el reactor con biomasa soportada sobre Pansil el fenómeno de inhibición es ligeramente inferior.

Por otro lado, con objeto de contrastar la validez del modelo de Levenspiel se calculan los valores teóricos de $K_{A}$ para cada digestor a partir de las ecuaciones (7) y (8), utilizando los valores medios de concentración de sustrato de cada experimento calculados a partir de los datos de la Tabla IV.

En las tablas $\mathrm{V}$ y $\mathrm{VI}$ se recogen los valores de la constante cinética, $\mathrm{K}_{\mathrm{A}}$, experimentales y teóricos así como el porcentaje de desviación entre ambos para cada concentración media de sustrato, en los digestores Testigo y con biomasa soportada sobre Pansil respectivamente. Como puede observarse, la desviación entre los valores teóricos y experimentales es inferior al $15 \%$ en todos los casos, llegando incluso a coincidir totalmente para algunos valores de concentración de sustrato; ello da idea de que las constantes cinéticas obtenidas mediante el modelo de Levenspiel, reproducen con bastante fidelidad el comportamiento de los reactores utilizados.
Tabla V

\begin{tabular}{|c|c|c|c|}
\hline$\underset{(g / l)}{s}$ & $\begin{array}{l}\text { KA exper. } \\
\left(\text { dias }^{-1}\right)\end{array}$ & $\begin{array}{l}K_{A \text { teorr. }} \\
\text { (dias-1) }\end{array}$ & \%Desviación \\
\hline 5,5 & 0,97 & 0,94 & 3 \\
\hline 6,4 & 0,50 & 0,50 & 0 \\
\hline 7,3 & 0,25 & 0,25 & 0 \\
\hline 7,8 & 0,16 & 0,16 & 0 \\
\hline 8,2 & 0,13 & 0,11 & 15 \\
\hline 8,6 & 0,09 & 0,08 & 11 \\
\hline
\end{tabular}

Valores experimentales y teóricos de la constante cinética aparente, $\mathrm{K}_{\mathrm{A}}\left(\right.$ días $\left.^{-1}\right)$, para cada concentración media de sustrato, $\mathbf{S}(\mathrm{g} / \mathrm{l})$, en el reactor Testigo

Tabla VI

\begin{tabular}{|c|c|c|c|}
\hline$\underset{(g / l)}{S}$ & $\begin{array}{l}K_{A \text { exper. }} \\
\left(\text { dias }^{-1}\right)\end{array}$ & $\begin{array}{l}\text { KA teór. } \\
\text { (dias }^{-1} \text { ) }\end{array}$ & \% Desviación \\
\hline 5,2 & 1,08 & 1,02 & 6 \\
\hline 6,0 & 0,58 & 0,60 & 3 \\
\hline 6,7 & 0,41 & 0,36 & 12 \\
\hline 7,3 & 0,26 & 0,23 & 12 \\
\hline 7,7 & 0,18 & 0,16 & 11 \\
\hline 8,2 & 0,13 & 0,11 & 15 \\
\hline
\end{tabular}

Valores experimentales y teóricos de la constante cinética aparente $K_{A}\left(\right.$ dias $\left.^{-1}\right)$, para cada concentración media de sustrato, $S(g / l)$, en el reactor con biomasa soportada sobre Pansil

Finalmente, dado que para cada experimento realizado se conoce el volumen de metano producido, así como las DQO inicial y final (Tablas II, III y IV) se calcula el coeficiente de rendimiento para ambos digestores conjuntamente, al no haberse encontrado diferencias significativas entre ambos; para ello se efectúa un ajuste lineal por mínimos cuadrados de las parejas de valores (G, DQO consumida) obteniéndose a través de la pendiente un valor de $315 \mathrm{ml} \mathrm{CH}_{4}$ en condiciones stándar de presión y temperatura (STP)/g DQO. Por otra parte, el porcentaje de reducción de DQO respecto a los valores añadidos es superior al $87 \%$ en ambos digestores, lo que da idea de la bondad del proceso anaerobio para eliminar la mayor parte de la DQO residual presente en el alpechín previamente tratado de forma aerobia.

\section{CONCLUSIONES}

A pesar de haber efectuado un tratamiento previo, vía aerobia con Geotrichum candidum, la digestión anaerobia del alpechín de almazara clásica de prensa, presenta una fuerte inhibición al aumentar la concentración de sustrato.

Se ha correlacionado la constante cinética aparente del proceso de digestión anaerobia con la concentración de sustrato mediante el modelo propuesto por Levenspiel. Los valores de las constantes de inhibición del modelo 
reproducen las constantes aparentes de velocidad del proceso anaerobio con un grado de desviación inferior al $15 \%$ en todos los casos.

Los valores obtenidos no permiten establecer diferencias significativas entre el comportamiento del digestor Testigo y del que tiene la biomasa soportada sobre Pansil, si bien en este último el grado de inhibición es ligeramente inferior.

La digestión anaerobia elimina del orden del $80 \%$ de la DQO, con un coeficiente de rendimiento de $315 \mathrm{ml}$ $\mathrm{CH}_{4}$ STP/g DQO.

\section{AGRADECIMIENTOS}

Queremos hacer constar nuestro agradecimiento a D. a Carmen Sánchez por su eficaz ayuda y colaboración.

\section{BIBLIOGRAFIA}

American Public Health Association (1985).- "Standard Methods for the Examination of Water and Wastewater".- (16th edn). Washington, DC.
Borja, R.; Martín, A. y Fiestas, J.A. (1990).- "Estudio cinético de la depuración anaerobia del alpechín en presencia de diversos soportes para la inmovilización de los microorganismos responsables del proceso".- Grasas y Aceites 41, 347-356.

De Caro, M. and Ligori, M.G. (1959). - "Activita antibiotica di un estratto desunto dalle acque di vegetazione delle olive".- Rend. Ist. Super Sanita 22, 223-243.

Fiestas, J.A.; Martín, A. and Borja, R. (1990).- "Influence of immobilization supports on the kinetic constants of anaerobic purification of olive mill wastewater".- Biological Wastes 33, 131-142.

Fiestas, J.A. y Borja, R. (1988).- "Aprovechamiento y depuración integral del alpechín".- Revista Técnica de Medio Ambiente 4, 97-100.

Grujer, W. and Zehnder, J.B. (1983).- "Conversion process in anaerobic digestión".- Wat. Sci. Tech. 15, 127-167.

Han, K. and Levenspiel, O. (1988).- "Extended monod kinetics for substrate, product and cell inhibition".- Biotechnology and Bioengineering 32, 430-437.

Ragazzi, E. and Veronesse, G. (1967).- "Recherche sulle fenolissidasi $\theta$ sul contenuto in o-difenoli delle olive".- Ann. Chim. 57, 1456-1492.

Rodriquez, M.; Pérez, J.; Ramos, A. and Martinez, J. (1988).- "Effect of extracts obtained from olive oil mill wastewaters on Bacilus megaterium ATCC 33085".- J. Appl. Bacteriol 64, 219-226.

Sorlini, C.: Andreoni, V.: Ferrari, A. y Ranalli, G. (1986).- "The influence of some phenolic acids present in oil-mill waters on microbic groups for the methanogenesis".- Internacional Symposium on Olive by Products Valorization. Sevilla, Spain. March, 223-243.

Time Series Processor (TSP) P.O. Box 61015, Station A. Palo Alto, 94306 CA. USA.

Winkler, M. (1983).- "Biological Treatment of Waste-water".- Ellis Horwood Ltd, Publishers. Chapter 4.

(Recibido: Agosto 1991) 